\title{
La structure des semiconducteurs fondus
}

\author{
J.-P. Gaspard et J.-Y. Raty \\ Physique de la Matière Condensée, Université de Liège, B5, 400 Sart-Tilman, Belgique
}

\begin{abstract}
Résumé : L'étude de la liaison chimique et son évolution sous conditions extrêmes de température et de pression est un challenge important de la physique de la matière condensée. Nous étudions les systèmes (éléments de groupes V, VI et VII)qui présentent une brisure spontanée de symétrie, la distorsion de Peierls, dans l'état cristallin et nous êtudions si cette distorsion persiste dans l'état liquide. Nous montrons que le terme répulsif joue un rôle important. Enfin, dans les composées IV-VI, GeSe et GeTe, nous montrons l'existence d'une transition de phase réentrante.
\end{abstract}

\section{INTRODUCTION : LA PROBLEMATIQUE}

L'étude de la liaison chimique dans la matière condensée est certainement un des problèmes les plus fondamentaux de la physique [1]. C'est la liaison chimique qui gouverne les propriétés de la cohésion (l'énergie totale), la structure (déterminée par diffusion élastique ou diffraction ${ }^{1}$ ) et les propriétés vibrationnelles (mesurées par diffusion en mode inélastique). Le but de ce chapitre est d'étudier, dans les grandes lignes, la cohésion entre les atomes dans les systèmes covalents et son évolution aux hautes températures et aux hautes pressions. Si les deux bases physiques de la cohésion, l'électromagnétisme et la mécanique quantique, sont parfaitement connues, le problème n'est cependant pas soluble en pratique.

L'interaction électromagnétique conditionne toute la chimie, la science des matériaux, les sciences du vivant et les sciences de la terre.

Dans le domaine des distances interatomiques $(\approx 2 \AA)$ et aux énergies mises en jeu, la mécanique quantique s'impose pour les électrons. La complexité mathématique du problème, $10^{24}$ électrons en interaction par $\mathrm{cm}^{3}$, est telle que, si on peut écrire les équations, on ne peut pas les résoudre! Il s'agit d'une limitation pratique et non fondamentale, mais limitation quand même ${ }^{2}$. Ceci impose un traitement séparé des différents types de liaisons chimiques, avec chaque fois de fortes approximations.

Nous allons analyser ici la liaison covalente dans les éléments des colonnes IV, V, VI et VII du tableau périodique et certains alliages.

\footnotetext{
1 Nous utiliserons ici le terme diffraction, même dans le cas des systèmes non cristallins.

2 A ce stade, il est intéressant de noter la foi que les anciens professaient à l'égard des progrès de la physique théorique. Lavoisier a déclaré : "Peut-être un jour la précision des données sera-t-elle amenée au point que le géomètre pourra calculer, dans son cabinet, les phénomènes d'une combinaison quelconque, pour ainsi dire de la même manière qu'il calcule le mowvement des corps célestes. Les vues que Monsieur de la Place a sur cet objet, \& les expériences que nous avons projetées, d'après ces idées, pour exprimer par des nombres la force des affinités des différens corps, permettent de ne pas regarder cette espérance comme une chimère".
}

Cette déclaration pourrait-elle encore être faite de nos jours, deux siècles plus tard? Il est intéressant de noter qu'à l'époque, les deux piliers de la description de la cohésion n'existaient pas : l'électricité était en gestation et la mécanique quantique ne nâttra qu'un bon siècle plus tard. 


\subsection{La règle de l'octet}

\begin{tabular}{|c|c|c|c|c|c|c|c|}
\hline I & II & III & IV & $\mathrm{V}$ & VI & VII & VIII \\
\hline alcalins & $\begin{array}{l}\text { alcalino- } \\
\text { terreux }\end{array}$ & terreux & carbonides & azotides & sulfurides & halogènes & gaz rares \\
\hline $\mathrm{s}$ & $\mathrm{s}^{2}$ & $\mathrm{~s}^{2} \mathrm{p}$ & $\mathrm{s}^{2} \mathrm{p}^{2}$ & $\mathrm{~s}^{2} \mathrm{p}^{3}$ & $\mathrm{~s}^{2} \mathrm{p}^{4}$ & $\mathrm{~s}^{2} \mathrm{p}^{5}$ & $\mathrm{~s}^{2} \mathrm{p}^{6}$ \\
\hline $\bar{H}$ & & & & & & & $\mathrm{He}$ \\
\hline $\mathbf{L i}$ & $\mathbf{B e}$ & B & $\mathbf{C}$ & $\mathbf{N}$ & $\mathbf{O}$ & $\mathbf{F}$ & $\mathrm{Ne}$ \\
\hline $\mathbf{N a}$ & $\mathbf{M g}$ & Al & $\mathbf{S i}$ & $\mathbf{P}$ & $\mathbf{S}$ & $\overline{\mathrm{Cl}}$ & $\mathbf{A r}$ \\
\hline $\mathbf{K}$ & $\mathrm{Ca}$ & Ga & Ge & As & Se & $\mathrm{Br}$ & $\mathbf{K r}$ \\
\hline $\mathbf{R b}$ & $\mathbf{S r}$ & In & Sn & $\mathbf{S b}$ & $\mathrm{Te}$ & I & $\mathrm{Xe}$ \\
\hline Cs & Ba & Tl & $\mathbf{P b}$ & $\mathbf{B i}$ & Po & At & $\mathbf{R n}$ \\
\hline \multicolumn{3}{|c|}{ structures compactes } & $\begin{array}{c}\text { ouverte } \\
\Downarrow \\
\text { compact. }\end{array}$ & \multicolumn{3}{|c|}{ structures ouvertes } & Compact \\
\hline Voisins & $8(+6)$ & ou 12 & $3 \Rightarrow 12$ & $3(+3)$ & $2(+4)$ & $1(+7)$ & 12 \\
\hline Structur & $3 \mathrm{D}$ & & $2 \mathrm{D}, 3 \mathrm{D}$ & $2 \mathrm{D}$ & $1 \mathrm{D}$ & OD & \\
\hline \multicolumn{3}{|c|}{ Liaisons: non directionnelles } & \multicolumn{4}{|c|}{ directionnelles } & non dir. \\
\hline \multicolumn{3}{|c|}{ Conduction: conducteurs } & \multicolumn{3}{|c|}{ semiconducteurs } & \multicolumn{2}{|c|}{ isolants } \\
\hline
\end{tabular}

Tableau 1. Tableau périodique (hormis les longues séries): structure, configuration électronique et liaisons chimiques des éléments.

Si la complexité du problème est déjà grande pour les systèmes cristallins, la situation est encore davantage compliquée pour les systèmes désordonnés (alliages, amorphes et liquides) qui ne possèdent plus d'éléments de symétrie qui permettent de réduire considérablement le nombre de paramètres. Cependant, malgré la complexité de la question, nous allons montrer qu'il est possible de trouver des comportements systématiques [2] et de proposer des modèles qualitatifs simples. L'analyse structurale par diffraction des neutrons apportent des informations importantes en ce domaine.

Dans les solides et liquides covalents (col. IV à VII), les atomes sont liés entre eux principalement par liaison $\mathrm{pp} \sigma$ des orbitales $\mathrm{p}$. Puisqu'il y a 6 lobes $\mathrm{p}$ par atome, on s'attend à avoir des structures à 6 voisins ${ }^{3}$. Cependant, l'observation des structures (cf. Tableau 1) montre que, dans les structures cristallines, au lieu d'avoir 6 premiers voisins, les atomes ont un nombre plus faible de voisins, résultat d'une instabilité électronique appelée la distorsion de Peierls [4]. Il s'agit d'un exemple de brisure spontanée de symétrie ou encore d'instabilité électronique.

Dans l'arsenic par exemple (figure 1 (a)), le cubique simple est déformé de manière à ce que les liaisons soient alternativement courtes (en gras) et longues (en traits fins).

\footnotetext{
${ }^{3}$ Seul le polonium cristallise dans la structure cubique simple.
} 


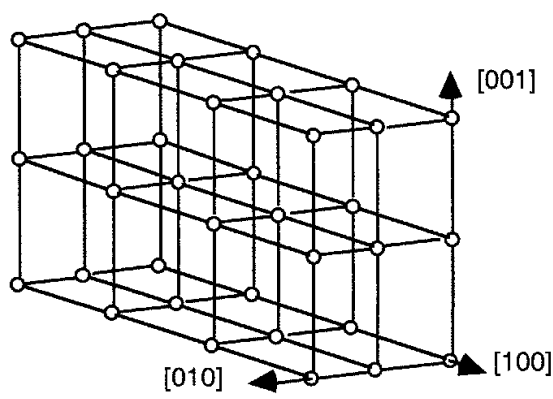

Cubique simple (c)

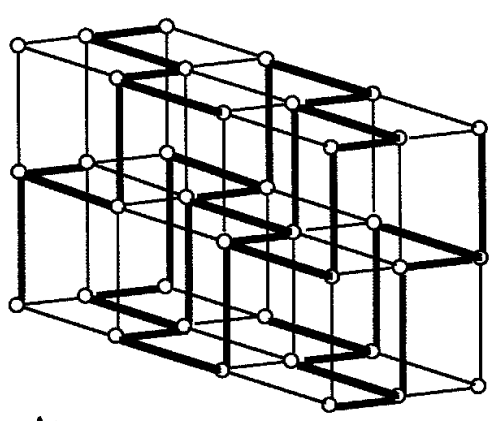

(a)

Figure 1. Structure cubique simple ((c), à gauche) et structure A7 de l'arsenic (schématisée, (a), à droite). Les trois liaisons covalentes, courtes, sont en gras tandis que les trois liaisons de Van der Waals, plus longues, sont notées en traits fins. On la note $3(+3)$. On voit que la structure A7 est formée de plans gaufrés empilés dans la direction $(1,1,1)$ du cubique simple.

Chaque atome est entouré de 3 voisins à courte distance $(2.5 \AA)$ et de trois voisins à plus grande distance $(3.1 \AA)$. De la même manière, dans la colonne VI, un atome a $2(+4)$ voisins tandis que dans la colonne VII, un atome a $1(+5)$ voisins.

Ceci constitue la règle de l'octet. Le nombre de voisins $\mathrm{Z}$ est égal à 8 moins le nombre d'électrons $\mathrm{s}$ et $\mathrm{p}\left(\mathrm{N}_{\mathrm{sp}}\right)$,

$$
Z=8-N_{s p}
$$

Cette règle est largement observée dans les systèmes monoatomiques à liaisons covalentes et également dans les composés et alliages. Dans ce cas, la règle de l'octet relie la coordinence moyenne, $\overline{\mathrm{Z}}$, au nombre moyen d'électrons s et $\mathrm{p}, N_{s p}{ }^{4}$.

Cette règle de l'octet est une idée reçue, adoptée et utilisée avec beaucoup de succès par les chimistes et les physiciens, sans vraie justification. Elle peut cependant se démonter assez simplement, au moins pour les colonnes V, VI et VII du tableau périodique [3]. Elle s'applique aux atomes liés par des orbitales p, directionnelles. Les structures étant assez ouvertes, les effets stériques sont peu importants et les effets électroniques déterminent la structure. La colonne $\mathrm{V}$ donne lieu à des plans d'atomes tricoordonnés, la colonne VI donne des anneaux $\left(\mathrm{S}_{8}\right)$ ou des chaînes ( $\mathrm{Se}, \mathrm{Te}$ ) d'atomes dicoordonnés tandis que les halogènes forment des cristaux moléculaires de molécules diatomiques.

La règle de l'octet souffre d'exceptions, en particulier les éléments du haut du tableau périodique $\left(\mathrm{N}_{2}, \mathrm{O}_{2}\right)$ forment des molécules diatomiques par effet de corrélation électronique. Dans le bas du tableau périodique, le polonium est cubique simple, donc avec 6 premiers voisins alors qu'il possède 6 électrons $s$ et $p$.

Nous allons montrer que la structure de plus haute symétrie n'est pas nécessairement celle de plus grande stabilité.

4 On notera que le nombre de liaisons longues est égal au nombre d'électrons p. 
L'intégrale de résonance, qui représente l'interaction quantique entre deux orbitales portées par deux atomes voisins, décroît avec la distance et cette décroissance peut être modélisée [5] selon une loi de puissance inverse

$$
\beta(r)=\frac{\beta_{0}}{r^{q}}
$$

le paramètre q est voisin de 2-3 pour les semiconducteurs et le facteur $\beta_{0}$ caractérise l'intensité de la résonance.

Le potentiel répulsif effectif peut être modélisé par

$$
V(r)=\frac{V_{0}}{r^{p}}
$$

$V_{0}$ caractérise l'intensité de la répulsion. Le paramètre $\mathrm{p}$ doit être supérieur à $\mathrm{q}$ sans quoi les atomes s'effondreraient sur eux-mêmes. Ces paramètres sont ajustés pour reproduire certaines valeurs expérimentales comme par exemple les paramètres de la maille cristalline, l'énergie de cohésion ou la compressibilité.

Plus le nombre de couches électroniques fermées est important, plus la valeur de $\mathrm{p}$ est élevée et plus l'énergie de la distorsion de Peierls est faible. On démontre [18] que le produit des deux paramètres $\mathrm{p}$ et $\mathrm{q}$ est donné par la formule

$$
p q=9 \frac{B}{\frac{E}{V}}
$$

où $\mathrm{B}$ est le module d'incompresssibilité et $\mathrm{E} / \mathrm{V}$ la densité d'énergie de cohésion (en module) par unité de volume.

De cette formule et de données expérimentales, on tire des valeurs du paramètre $\mathrm{p}$ (tableau 2).

\begin{tabular}{lllllll}
\hline & $\mathrm{r} / \mathrm{r}_{\mathrm{v}}$ & $\begin{array}{l}\mathrm{E} \\
\mathrm{kJ} / \text { mole }\end{array}$ & $\begin{array}{l}\mathrm{V} \\
\mathrm{cm}^{3}\end{array}$ & $\begin{array}{l}\mathrm{E} / \mathrm{V} \\
\mathrm{kJ} / \mathrm{cm}^{3}\end{array}$ & $\begin{array}{l}\mathrm{B}^{-1} \\
\mathrm{~cm}^{3} / \text { dyne }\end{array}$ & $\mathrm{p}$ \\
\hline $\mathrm{As}$ & 1.25 & 285 & 13.1 & 21.8 & 2.54 & $\mathbf{5 . 4}$ \\
$\mathrm{Sb}$ & 1.17 & 265 & 18.4 & 14.4 & 2.61 & $\mathbf{8 . 0}$ \\
$\mathrm{Bi}$ & 1.12 & 210 & 21.3 & 9.9 & 3.17 & $\mathbf{9 . 6}$ \\
\hline
\end{tabular}

Tableau 2. Valeurs de la distorsion, de la densité d'énergie de cohésion $E / V$, de l'incompressibilité $\mathrm{B}^{-1}$ et du paramètre $\mathrm{p}$ pour les éléments de la colonne $\mathrm{V}$. Les données sont rapportées à une mole. La valeur de q est égale à 2 .

Les systèmes covalents subissent souvent une brisure spontanée de symétrie, qui est une instabilité électronique, appelée distorsion de Peierls. Elle est l'analogue, pour les systèmes étendus, de la distorsion de Jahn-Teller pour les systèmes finis (molécules ou impureté dans un cristal). Dans 
l'effet Jahn-Teller, un niveau dégénéré et partiellement occupé donne lieu à une diminution de la symétrie qui lève la dégénérescence et induit un gain d'énergie. Dans les systèmes étendus, c'est l'ouverture d'une bande interdite au niveau de Fermi qui assure un gain d'énergie de cohésion. Le moteur énergétique de la distorsion de Peierls [4] est décrit sur la figure 2.

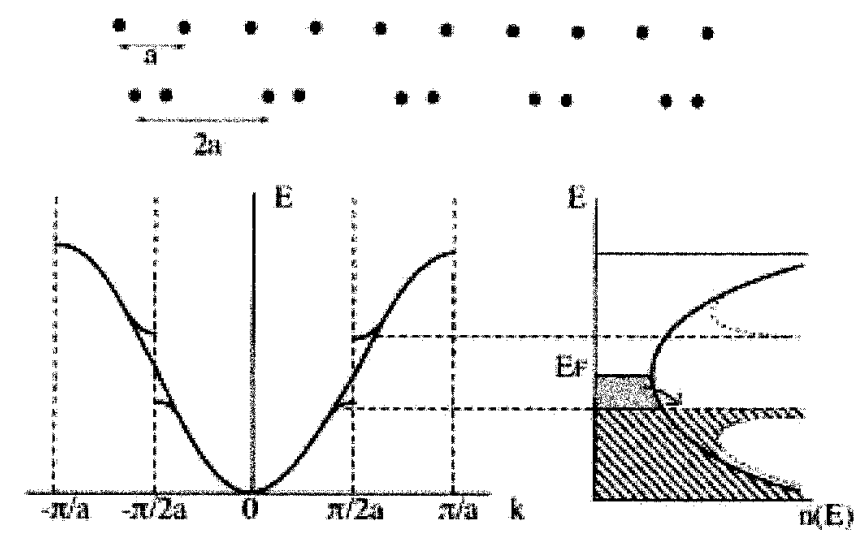

Figure 2. Relations de dispersion et densités d'états $n(E)$ d'une chaîne linéaire. En haut, chaîne non distordue et métallique (le niveau de Fermi est au milieu de la bande). En dessous, chaîne distordue par doublement de la maille devenue isolante (ou semiconductrice). Lorsque la maille passe de a à $2 \mathrm{a}$, la zone de Brillouin est réduite de moitié. On observe que la distorsion déplace une bande d'états occupés (grisés) juste sous le niveau de Fermi $\mathrm{E}_{\mathrm{F}}$ vers des plus basses énergies.lors de l'ouverture de la bande interdite, ce qui stabilise la structure distordue.

La distorsion de la figure 1 double la maille dans chacune des directions $\mathrm{a}$, $\mathrm{b}$ et $\mathrm{c}$ de l'espace. Une bande interdite s'ouvre alors au milieu de la bande. Si le niveau de Fermi est au milieu de la bande niveau de Fermi, il s'ensuit un gain d'énergie cohésive. En effet, les états proches du niveau de Fermi sont déplacés vers des régions plus basses (liantes) en énergie, d'où un gain d'énergie électronique. Dans le cas non distordu, le niveau de Fermi est situé dans une bande et le système est métallique. Dans le cas distordu, la structure est semiconductrice ou isolante si la distorsion est forte.

Cependant, ce gain d'énergie électronique est contrebalancé par une énergie de répulsion qui s'oppose à la distorsion. Nous allons voir que cette énergie de répulsion joue un rôle important et peut inhiber la distorsion.

L'énergie de la liaison covalente est proportionnelle à la racine carrée $\sqrt{Z}$ de la coordinence $Z$, En effet, dans la liaison covalente, la cohésion est due à l'élargissement en bande d'un niveau atomique partiellement occupé. L'élargissement est un écart-type dont la variance est égale à $Z \beta^{\leftarrow}$. L'énergie de répulsion est proportionnelle au nombre de premiers voisins. L'énergie totale s'écrit donc

5 C'est une conséquence du théorème des moments. Voir p. ex. Elliott S. The physics and chemistry of solids, J. Wiley and Sons, 1998. 


$$
E_{t} \propto-\sqrt{Z} \frac{\beta_{0}}{r^{q}}+Z \frac{V_{0}}{r^{p}}
$$

A la distance d'équilibre, l'énergie totale varie comme:

$$
E_{t} \propto Z^{\frac{p-2 q}{2(p-q)}}
$$

Dans le cadre de ce modèle, simple, on trouve que la structure la plus stable est soit

a) une structure compacte, cubique et métallique si $p>2 q$

b) une structure de faible coordinence $(Z=1)$ de molécules diatomiques si $p<2 q$.

La colonne I du tableau périodique illustre bien ce comportement. L'hydrogène qui n'a pas de couche fermée et qui est donc très compressible (faible rapport $\mathrm{p} / \mathrm{q}$ ) se structure en molécules diatomiques tandis que les éléments plus lourds $(\mathrm{Li}, \mathrm{Na}, \mathrm{K}, .$.$) donnent lieu à des phases$ compactes.

L'instabilité électronique (et le taux de distorsion) est donc reliée à la dureté du potentiel répulsif entre atomes (paramètre $p$ ). Puisque $p>q$ pour assurer la stabilité de la matière, on voit donc que, lorsque le potentiel est fortement répulsif $(p>2 q)$, les phases de hautes coordinences (CFC, HC) sont favorisées tandis que lorsque le potentiel répulsif est "mou", les faibles coordinences sont favorisées (p. ex. molécules diatomiques $\mathrm{H}_{2}, \mathrm{~N}_{2}, \mathrm{O}_{2}, .$. ). Dans le cas limite des sphères dures $(p=\infty)$ il ne peut y avoir de distorsion.

Dans ce modèle schématique piloté par la formule (6), on a soit une structure métallique compacte soit une structure moléculaire isolante (ou semiconductrice).

Un traitement plus élaboré, tenant compte de la directionnalité des orbitales $\mathrm{p}$ et du nombre d'électrons p, conduit à la règle de l'octet (équation (1)) [3]. En effet, pour gagner de l'énergie de cohésion, il faut ouvrir une bande interdite au niveau de Fermi et donc il faut adapter la distorsion en conséquence. C'est ainsi que le doublement de la longueur de la maille dans les trois directions de l'espace par alternance de liaisons courtes et longues CLCLCL ouvre une bande interdite au milieu de la bande et donc stabilise les éléments qui ont une bande p demi-remplie (colonne $\mathrm{V}$ : $\mathrm{AS}, \mathrm{Sb}, \mathrm{Bi})$ ). Dans le cas d'une bande $2 / 3$ remplie (colonne VI), le triplement de la maille CLLCLLCLL ouvre un gap au niveau de Fermi, situé au 2/3 de la bande. En général, dans le cas d'une bande $\mathrm{m} / \mathrm{n}$ remplie ( $\mathrm{m}$ et $\mathrm{n}$ entiers premiers) remplie, la maille est multipliée par $\mathrm{n}$ dans chacune des trois directions de l'espace. En utilisant certains arguments simples, on peut montrer que la distorsion de Peierls peut aussi s'expliquer en se basant sur un formalisme purement local, à savoir la méthode des moments appliquée à des potentiels de liaisons fortes [3], et est susceptible d' exister en l'absence de symétrie de translation.

La question est de savoir si la règle de l'octet est aussi observée dans les liquides et aux hautes pressions. La diffraction des neutrons permet de résoudre cette question. Pour ce faire, nous avons entrepris une étude systématique des covalents élémentaires et leurs composés binaires [2].

\section{LIQUIDES}

\subsection{Un petit modèle physique de la fusion des cristaux}

L'état liquide, plus désordonné que l'état cristallin, a une entropie plus élevée que celle du cristal; 
par contre, il a une énergie de cohésion plus faible ${ }^{6}$ (en module) car les atomes sont moins bien "rangés" (certaines liaisons chimiques sont coupées ou plus faibles) que dans le cristal, périodique. L'énergie libre

$$
F=U-T S
$$

où $\mathrm{S}$ est l'entropie, doit être minimale à l'équilibre thermodynamique.

A basse température, l'état le plus stable est l'état cristallin ( $\mathrm{U}=\mathrm{E}_{\text {cohésion }}<0$ est minimum) tandis qu'à haute température, l'état le plus désordonné stable devient plus stable pour des raisons entropiques.

$$
\begin{aligned}
& \left|\mathrm{U}_{\text {cristal }}\right|>\left|\mathrm{U}_{\text {liquide }}\right| \\
& \mathrm{S}_{\text {cristal }}<\mathrm{S}_{\text {liquide }}
\end{aligned}
$$

L'entropie de configuration du liquide, désordonné, est beaucoup plus élevée que celle đu cristal, tandis que l'entropie de vibration du liquide est assez voisine de celle du cristal à une même température. Nous supposerons, en première approximation, que ces fonctions d'état $U$ et $S$ ne dépendent pas de la température. Pour simplifier davantage, on pourrait même poser, en première approximation, que $S_{\text {cristal }}=0$.

On peut donc représenter les énergies libres du cristal et du liquide calculées dans toute la gamme des températures comme montré sur la figure 3 .

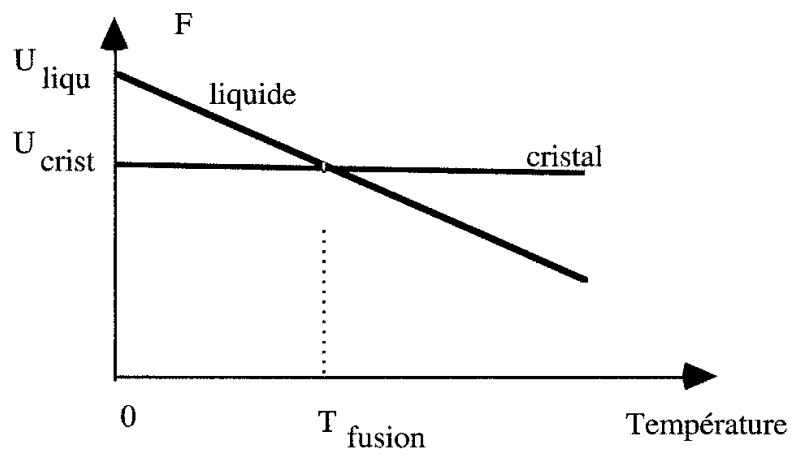

Figure 3. Energies libres F du liquide et cristal.

On voit donc qu'il existe une température, $\mathrm{T}_{\text {fusion }}$ au-dessus de laquelle la phase désordonnée (le liquide) est thermodynamiquement la plus stable.

\footnotetext{
6 En fait, il n'a jamais été démontré de manière générale que le cristal est plus stable qu'une phase amorphe de même composition.

$7 \mathrm{Si}$ on travaille à pression constante, c'est l'énergie libre de Gibbs $\mathrm{G}=\mathrm{F}+\mathrm{pV}$ qui doit être minimum. La différence n'est (en général) pas très importante pour les phases condensées.
} 
Dans ce modèle simple, la température de fusion est donnée par

$$
\mathrm{T}_{\text {fusion }}=\Delta \mathrm{U} / \mathrm{S}_{\text {fusion }}
$$

En première approximation, on peut supposer que, lors de la fusion, l'énergie de cohésion diminue de l'ordre de 2 à $4 \%$ tandis que l'entropie du liquide est de l'ordre de 2 cal. par atome-gr. pour les liquides normaux. Les systèmes covalents $(\mathrm{Si}, \mathrm{Ge}, \mathrm{Sb}$ ) s'écartent notablement de cette gamme, avec une entropie de fusion 3 fois plus élevée qui est liée au changement important de structure lors de la fusion [6].

\begin{tabular}{|l|c|c|c|c|c|c|c|c|c|}
\hline élément & $\mathrm{Ar}$ & $\mathrm{Na}$ & $\mathrm{Fe}$ & $\mathrm{Ni}$ & $\mathrm{Cu}$ & $\mathrm{Si}$ & $\mathrm{Ge}$ & $\mathrm{As}$ & $\mathrm{Sb}$ \\
\hline $\mathrm{U}$ (kcal/at.gr) & 1.85 & 26.0 & 98.9 & 102.3 & 80.8 & 107 & 89.3 & 69 & 62 \\
\hline $\mathrm{T}_{\mathrm{f}}(\mathrm{K})$ & 84 & 371 & 1809 & 1726 & 1356 & 1683 & 1210 & 1090 & 923 \\
\hline $\mathrm{S}_{\mathrm{f}}(\mathrm{cal} / \% / \mathrm{at.gr})$ & - & 1.7 & 2.0 & 2.4 & 2.3 & 6.6 & 6.7 & - & 5.3 \\
\hline$\Delta \mathrm{U} / \mathrm{U}(\%)$ & - & 2.4 & 3.8 & 4.0 & 3.9 & 10.4 & 9.1 & - & 7.9 \\
\hline
\end{tabular}

Tableau 3. Energies de cohésion U (en kcal par atome-gr), températures, entropies de fusion et variation relative de l'énergie de cohésion $\Delta U / U$ pour quelques éléments représentatifs des grandes familles.

Au plan théorique, la connaissance des liquides et du mécanisme de la fusion demande de connaître aussi bien le terme énergétique que le terme entropique puisque ces deux termes (U et TS) sont comparables au point de fusion et en son voisinage [6].

La physique des liquides est, à ce point de vue, fort compliquée car aucun de ces deux termes n'est calculable exactement. Signalons aussi l'aspect de moyenne temporelle sur les configurations et sur les contributions inélastiques de la diffraction par le liquide qui est un facteur supplémentaire de complexité. Si on ajoute à cela que les spectroscopies des liquides donnent des informations moins riches que dans le cas des cristaux, on mesure la difficulté de l'étude des liquides et la nécessité de combiner différentes techniques.

\subsection{Structure des liquides: Ordres de grandeur}

Les liquides présentent un ordre à courte distance mais ne présentant pas d'ordre à longue distance comme les cristaux et les quasicristaux. Le tableau 4 donne quelques ordres de grandeur des paramètres structuraux des liquides. Rappelons que le module du vecteur de diffusion $|\mathbf{q}|$ est relié à l'angle de diffraction par

$$
q=\frac{4 \pi}{\lambda} \sin (\theta)
$$

et s'exprime donc en $\AA^{-1}$ (ou en $\mathrm{nm}^{-1}$ ). 


\begin{tabular}{|l|c|c|c|c|}
\hline & & solide & liquide & \\
\hline $\begin{array}{l}\text { Distance des premiers } \\
\text { voisins: }(\AA)\end{array}$ & $\mathrm{R}_{1}$ & $2-3$ & $2-3$ & \\
\hline $\begin{array}{l}\text { Position du 1er pic de } \\
\text { diffraction: }\left(\AA^{-1}\right)\end{array}$ & $\mathrm{q}_{1}$ & $2.3-3.5$ & $2.3-3.5$ & $\approx 7.4 / \mathrm{R}_{1}$ \\
\hline $\begin{array}{l}\text { Largeur du premier pic } \\
\left(\AA^{-1}\right)\end{array}$ & $\Delta \mathrm{q}_{1}$ & $10^{-4}$ & 5 & \\
\hline $\begin{array}{l}\mathrm{L} \text { o n g u e u r } \\
\text { corrélation } 8(\AA)\end{array}$ & $\mathrm{L}$ & 60000 & 12 & $\approx 2 \pi / \Delta \mathrm{q}_{1}$ \\
\hline $\begin{array}{l}\text { Longueur de corrélation } \\
\text { (nombre de couches) }\end{array}$ & $\mathrm{L} / \mathrm{R}_{1}$ & 20000 & $3-4$ & $\approx 2 \pi /\left(\Delta \mathrm{q}_{1}\right.$ \\
\hline
\end{tabular}

Tableau 4. Ordres de grandeur des paramètres structuraux des liquides. Les largeurs de pics mentionnées et leurs quantités dérivées sont intrinsèques dans le cas des liquides tandis que pour les crist On voit donc que, dans le liquide, l'ordre des atomes dans les couches concentriques autour d'un atome s'étend sur une bonne dizaine d'angstroms, soir sur 3 à 4 couches atomiques. Au-delà, la fonction de corrélation de paires est égale à l'unité comme dans un gaz (voir figure 6) aux elles dépendent de la résolution expérimentale de l'instrument.

\subsection{Les trois voies d'étude des liquides}

La connaissance des liquides, systèmes assez complexes au plan structural, ne peut se faire qu'en combinant trois voies d'étude développées ci-après:

a) La diffraction des neutrons, des rayons $\mathrm{X}$ ou l'EXAFS (absorption des rayons $\mathrm{X}$ ) 9

b) La modélisation théorique de l'énergie et de l'entropie

c) La simulation sur ordinateur (Monte-Carlo et dynamique moléculaire)

\section{LA DIFFRACTION DES NEUTRONS (ET DES RAYONS X) [20]}

La principale information expérimentale sur la structure provient de la diffusion d'un rayonnement dont la longueur d'onde est comparable à la distance interatomique. Si, comme c'est le cas de la diffraction des rayons $\mathrm{X}$ ou des neutrons par les cristaux, un spectre de diffraction permet d'obtenir les paramètres de maille et de résoudre complètement la structure, il n'en va pas de même dans les liquides car l'absence de symétrie de translation implique que

I. Le nombre de paramètres est considérable (coordonnées de tous les atomes) puisqu'il ne peut être réduit du fait de l'absence de symétrie.

II. Le spectre de diffusion (diffractogramme) est peu structuré, donc pauvre en information, au plus peut-on en extraire une dizaine de paramètres.

Cependant, on peut obtenir des informations essentielles sur la liaison chimique (distances, nombre de premiers voisins). La combinaison avec d'autres techniques est indispensable et permet d'affiner ces informations.

Les neutrons sont particulièrement bien adaptés à l'étude des liquides, essentiellement pour des raisons pratiques. En effet, les neutrons ont une longueur de pénétration importante dans la matière

\footnotetext{
8 Obtenue par la formule de Scherrer

9 Extended X-ray Absorption Fine Spectroscopy
} 
et on peut donc étudier des échantillons d'un volume de l'ordre de quelques $\mathrm{cm}^{3}$. Pour la même raison, le conteneur, souvent de la silice amorphe, le four ou le cryostat n'atténuent pas le signal de manière rédhibitoire.

Le volume de cohérence des neutrons est aussi bien adapté à l'étude des liquides.

La longueur de cohérence longitudinale est

$$
L_{\text {long }}^{c o h}=\frac{1}{2} \frac{\lambda^{2}}{\Delta \lambda}
$$

où $\Delta \lambda$ est la largeur de la distribution des longueurs d'ondes autour de leur valeur moyenne $\lambda$ tandis que la longueur de cohérence transversale est donnée par [19]

$$
L_{\text {trans }}^{\text {coh }}=\frac{1}{2} \frac{\lambda}{\Delta \vartheta}
$$

où $\Delta \theta$ est la divergence angulaire du faisceau (rapport de la taille de la source à la distance sourceéchantillon). Le volume de cohérence des neutrons est bien adapté aux liquides et les verres car il est assez grand pour comprendre suffisamment d'atomes et ainsi représenter la structure globale, mais aussi assez petit pour qu'il y ait de nombreux volumes de cohérence dans l'échantillon, fournissant donc une bonne moyenne d'ensemble.

Actuellement les synchrotrons de $3^{\mathrm{c}}$ génération permettent d'obtenir des spectres de qualité, comparables à ceux des neutrons, en des temps très brefs. En effet, on peut y produire des rayons $\mathrm{X}$ de très haute brillance, de courte longueur d'onde et très pénétrants.

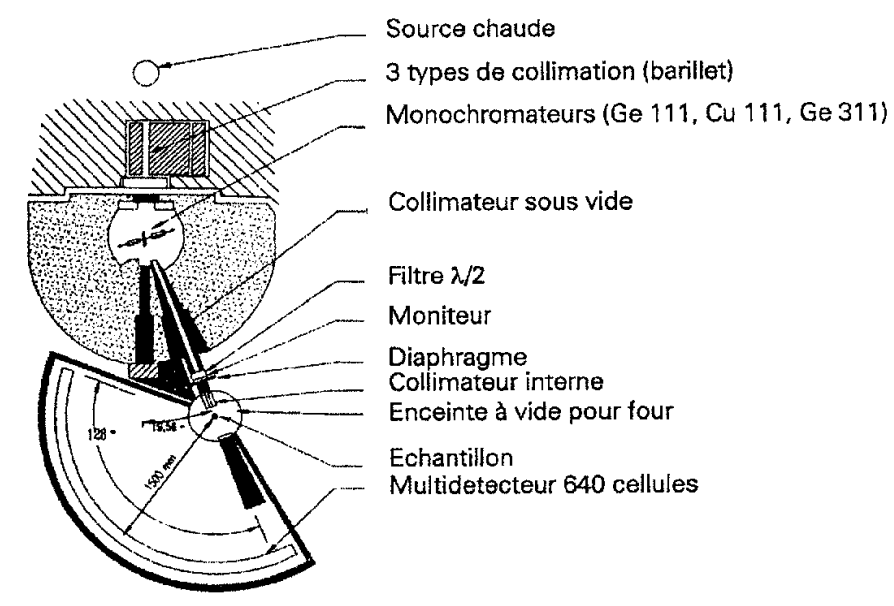

Figure 4. Schéma du diffractomètre de neutrons 7C2 (Laboratoire Léon Brillouin, Saclay) installé sur la source chaude et dédicacé à l'étude des liquides et amorphes. Un multidétecteur de 640 cellules couvre un champ de $128^{\circ}$. 
Historiquement, les premières études sur les liquides ont été réalisées à l'aide d'un diffractomètre de rayons X. Après le développement des réacteurs à faisceaux de neutrons, la majorité des études sur la structure des liquides y ont été réalisées, principalement sur les réacteurs qui disposent d'une source chaude [7]. Il s'agit d'un bloc de graphite élevé à haute température à l'aide du rayonnement $\gamma$ du cour, à une température de l'ordre de $2000 \mathrm{~K}$. Le spectre de longueurs est alors centré sur $\lambda=$ $0.7 \AA$. Les valeurs de $\mathrm{q}_{\max }=4 \pi / \lambda$ sont données dans le tableau 5 .

\begin{tabular}{|l|c|c|c|}
\hline source & Température $(\mathrm{K})$ & $\lambda(\AA)$ & $\mathrm{q}_{\max }\left(\AA^{-1}\right)$ \\
\hline thermique & 300 & 1.7 & 7.5 \\
\hline chaude (LLB) & 1400 & .7 & 15 \\
\hline chaude (ILL) & 2300 & 0.5 & 23 \\
\hline
\end{tabular}

Tableau 5. Longueurs d'ondes et valeurs maximales du vecteur de transfert q pour différentes sources pour $\lambda_{\max }$, en tenant compte des angles de diffraction maximaux (ILL: $2 \theta_{\max }=140^{\circ}$, LLB: $2 \theta_{\max }=128^{\circ}$ ).

Comme le facteur de structure d'un liquide a en général des oscillations jusque $15 \AA^{-1}$, le recours à une source chaude est indispensable. La source chaude de l'ILL permet même de descendre à 0.35 $\AA$ en gardant encore un flux acceptable et donc d'aller, en théorie, jusque $33 \AA^{-1}$ dans l'espace réciproque.

Les sources à spallation de neutrons (ISIS, UK et PSI, Suisse) sont aussi très bien adaptées à l'étude des liquides car elles produisent des neutrons de petites longueurs d'ondes.

Les neutrons sont bien adaptés à l'étude des liquides car:

(a) de bonnes données peuvent être obtenues jusque des valeurs élevées en $\mathrm{q}\left(16 \AA^{-1}\right)$ sans être atténuées par le facteur de forme atomique, celui du noyau étant totalement négligeable.

(b) l'environnement échantillon peut être géré de manière confortable, en effet:

I. Le conteneur est réalisé en silice $\left(\mathrm{SiO}_{2}\right)$, en vanadium ou en alumine (diamètre interne/externe typique: $6 \mathrm{~mm} / 8 \mathrm{~mm}$ )

II. Le four, une feuille de vanadium produisant la chaleur par effet Joule, diffuse de manière pratiquement isotrope ${ }^{10}$.

III. D'éventuels réflecteurs thermiques sont placés pour des expériences à très hautes températures $\left(\mathrm{T}>1000^{\circ} \mathrm{C}\right)$.

IV. On peut placer une cellule en NiZr pour expériences sous hautes pressions (jusque $1 \mathrm{GPa}$ ) sans altérer le faisceau.

V. On peut ainsi balayer une gamme de températures allant de la température ambiante (ou $1.5 \mathrm{~K}$ avec un cryostat) jusqu'à $1500^{\circ} \mathrm{C}$.

La figure 4 est un schéma du diffractomètre 7C2 [8] du Laboratoire Léon Brillouin (Saclay) qui est installé sur la source chaude. Il est analogue à un diffractomètre de poudre de faible résolution $\left(0.2^{\circ}\right.$ en $2 \theta$ ou $0.03 \AA^{-1}$ en q) dédié à l'étude des liquides et amorphes ${ }^{1}$. Un multidétecteur de 640 cellules couvre un champ de $128^{\circ}$. Celui-ci permet une mesure simultanée de toutes les valeurs de

10 En effet, la section efficace cohérente de diffusion du vanadium est pratiquement annulée par des concentrations très voisines d'isotopes de spin qui ont des sections efficaces opposées.

11 Puisque les spectres d'amorphes et liquides n'ont pas de structure en "pics de Bragg", on privilégie le flux à la résolution. 
$q$ et s'affranchit donc des variations temporelles du flux du réacteur ou des modifications de l'échantillon. Par contre, il faut calibrer l'efficacité des détecteurs, ce qui se fait à l'aide d'un barreau de vanadium qui, en théorie diffuse de manière isotrope.

Une expérience typique se conduit comme suit:

1. Mesure du signal sans échantillon (mesure des bruits électronique, des rayonnements parasites)

2. Mesure du signal de l'environnement échantillon (four p. ex.)

3. Mesure du signal de l'environnement échantillon et du conteneur vide

4. Mesure du signal de l'échantillon dans son environnement

5. Mesure d'un signal isotrope (p. ex. vanadium) pour correction d'efficacité des détecteurs

Des formules combinant ces différents spectres permettent d'extraire le signal provenant uniquement de l'échantillon.

\section{LA SIMULATION SUR ORDINATEUR}

La simulation sur ordinateur permet de s'affranchir d'un modèle d'entropie. Elle a apporté une aide considérable à notre compréhension des liquides et son développement est contemporain de celui des ordinateurs. En effet, les modèles d'entropie des liquides sont très imparfaits et l'obtention des fonctions de corrélation de paires même dans le cas de systèmes simples (p. ex. gaz rares) par résolution des équations intégrales (Percus-Yevick p. ex.) ne donne pas de résultats exacts. Dans le cas de liquides covalents, la mission est impossible.

Il a deux grands types de méthodes de simulation de systèmes désordonnés sur ordinateur :

- $\quad$ la méthode de Monte-Carlo [9] et

- la dynamique moléculaire [10].

La première calcule les propriétés moyennes à l'équilibre thermodynamique, de même que la seconde qui, en plus, permet d'étudier l'évolution temporelle du système, et donc de calculer des propriétés de transport.

Nous renvoyons le lecteur à des références classiques sur ces sujets $[9,10]$.

Nous montrerons, dans le cas des alliages IV-VI, comment la simulation sur ordinateur permet d'interpréter et de compléter les données de diffraction neutronique.

\section{FUSION DES SYSTEMES COVALENTS}

La fusion des semiconducteurs [11] se classe en deux grandes catégories :

a) les semiconducteurs du groupes IV et leurs composés isoélectroniques

b) les semiconducteurs des groupes V, VI et VII et leurs composés isoélectroniques

Dans le premier cas, l'environnement atomique passe d'une coordinence 4 (environnement tétraédrique) dans le cristal à un environnement de coordinence 6-7 dans le liquide (avec quelques exceptions que nous expliquerons).

Dans le second cas, la distorsion de Peierls se conserve ou disparaît, selon les énergies et entropies mises en jeu.

\subsection{La fusion des semiconducteurs du groupe IV et composés III-V}

Les semiconducteurs de la colonne IV et leurs composés isoélectroniques III-V fondent en changeant de coordinence, principalement pour des raisons entropiques. Leur changement de 


\section{NEUTRONS ET SYSTÈMES DÉSORDONNÉS}

structure est semblable à la transition de $\alpha$-Sn $(Z=4)$ à $\beta-S n(Z=4+2)$ de l'étain. En effet, les semiconducteurs élémentaires $\mathrm{Si}$ et Ge et les composés GaAs, InAs, InSb,.. fondent en adoptant une coordinence de $Z \approx 6.5$ dans le liquide alors qu'elle est de $Z=4$ dans le cristal (diamant, blende ou würtzite). Cette compactification de l'environnement atomique local se traduit par une augmentation de la densité lors de la fusion alors que la distance interatomique augmente. La conductivité électrique passe de celle d'un semiconducteur à celle d'un métal. L'entropie de fusion est énorme, environ trois fois celle des métaux normaux ou gaz rares. En effet, l'entropie des structures à coordinence 6 est assez élevée car six voisins autour d'un atome central ne constitue pas une couche complète et laisse donc plus d'espace de liberté qu'une structure compacte, de coordinence 12. A l'opposé, les structures à coordinence tétraédriques ont une grande rigidité angulaire qui diminue leur entropie. Ceci est évident sur la fonction de corrélation de paires g(r) qui atteint plus rapidement la valeur asymptotique de 1 que pour les structures liquides tétracoordonnées ou de haute coordinence (10 à 12). Enfin, le spectre de diffraction est assez peu courant: le rapport des deux premiers maxima $\mathrm{q}_{2} / \mathrm{q}_{1}$ du spectre de diffraction est égal ou supérieur à 2 , ce qui est une valeur exceptionnellement élevée. Ces valeurs sont mentionnées dans le tableau 6.

\subsection{Les composés II-VI}

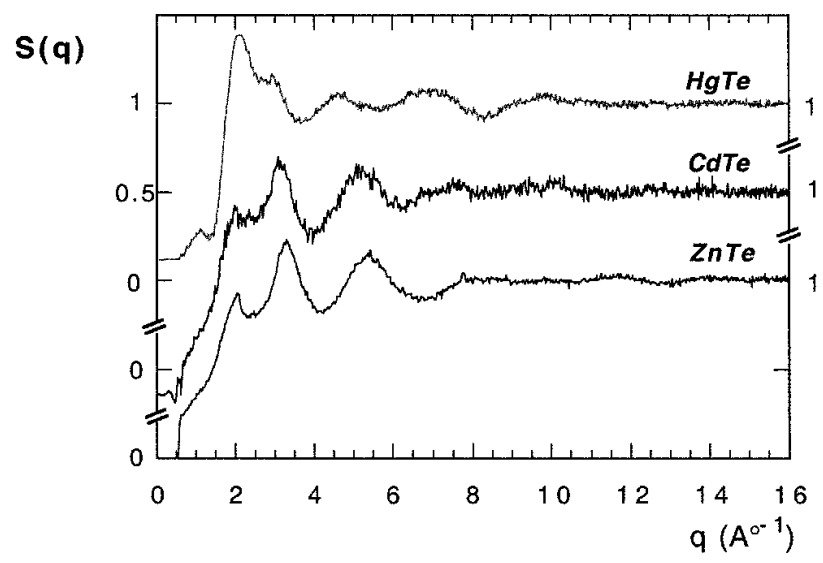

Figure 5. Spectre de diffraction des semiconducteurs II-VI à base de Te : ZnTe, CdTe et HgTe. On observe que les rapports d'intensité des deux premiers pics s'inverse lorsque l'on passe de ZnTe et CdTe à $\mathrm{HgTe}$. Un premier pic plus élevé correspond davantage à un état métallique, plus compact.A l'opposé des précédents, ils ne changent généralement pas de coordinence lors de la fusion, sauf les plus lourds comme $\mathrm{HgTe}$. Composés de métaux divalents $(\mathrm{Zn}, \mathrm{Cd}, \mathrm{Hg}$ ) du groupe IIb et de chalcogènes ( $\mathrm{S}, \mathrm{Se}, \mathrm{Te}$ ) du groupe VI, ils sont importants dans le domaine de l'optó́lectronique: les lasers et détecteurs (dans l'infrarouge et dans le visible) promis au plus grand avenir.

La fusion de $\mathrm{ZnTe}$, dont la structure blende est stable dans un grand domaine de pression, se produit à $1290^{\circ} \mathrm{C}$ et l'entropie de fusion est plus faible que pour les semiconducteurs élémentaires 
(3.9 kcal/at.g.K contre 7.1 cal/at.g.K pour Si). A la fusion, la conductivité électrique de ZnTe passe de $10 \Omega^{-1} \cdot \mathrm{cm}^{-1}$ dans le solide à $50 \Omega^{-1} \cdot \mathrm{cm}^{-1}$ dans le liquide avant de croître plus lentement par la suite: c'est la caractéristique d'une fusion de type semiconducteur-semiconducteur, contrairement au Si, Ge et autres composés III-V, où la fusion est de type semiconducteur-métal.

De façon originale, l'ordre des semiconducteurs liquides CdTe, $\mathrm{ZnTe}$ et $\mathrm{HgSe}$ est tétraédrique, et la liaison chimique est de type $\mathrm{sp}^{3}$. Il n'y a donc pas de changement important de la nature de la liaison chimique lors de la fusion. Par contre, la fusion de HgTe s'accompagne, comme pour la grande majorité des semiconducteurs connus, d'un changement radical d'ordre local qui devient octaédrique déformé dans le liquide, et présente une transition vers un état métallique. Remarquons également que pour ces composés, l'effet de la température et celui de la pression sont similaires [12]. Le cas de HgS liquide est particulier: qualitativement, il présente une structure en chaînes, comparable à celle du sélénium ou du soufre liquides.

\subsection{La fusion des semiconducteurs des groupes V et VI}

Les semiconducteurs de la colonne $\mathrm{V}$ sont des exemples classiques d'éléments dont la structure cristalline dérive d'une distorsion de Peierls d'une structure cubique simple [1]. La transition géométrique que nous avons décrite sur la figure 1 génère une bande interdite au niveau de Fermi et stabilise ainsi la structure A7 des éléments du groupe Va: As Sb et Bi. Nous ne considérerons ici que l'As et les composés d'As et $\mathrm{Sb}$ qui sont représentatifs de ces systèmes qui possèdent 5 électrons de valence par atome et sont donc tricoordonnés suivant la règle de l'octet (1). Selon la dureté du terme répulsif (le paramètre $\mathrm{p}$ de l'équation (3)) et la température de fusion, la distorsion de Peierls est encore présente dans le liquide ou a disparu. La transition est d'autant plus prononcée que le rapport $\mathrm{p} / \mathrm{q}$ est faible.

Pour l'arsenic, la distorsion de Peierls peut subsister au-delà du point de fusion, à haute température $\left(\mathrm{T}=1100^{\circ} \mathrm{C}\right.$ pour $\left.\mathrm{As}\right)$. En effet, il a été montré par diffraction de neutrons que l'ordre local de l'arsenic liquide, très voisin de celui du cristal, évolue peu avec l'augmentation de la température [13]. La structure de liquide est due à la persistance d'une distorsion de Peierls au niveau de l'ordre local. Celle-ci induit la présence d'un creux (ou pseudo-gap, voire même un gap comme sur la figure 2) au niveau de Fermi du liquide, ce qui stabilise un environnement atomique local dans lequel les 6 voisins d'un atome du liquide se situent aux sommets d'un octaèdre distordu (3 liaisons courtes et 3 trois liaisons longues dans le cas de As) et confère à l'arsenic liquide un caractère semiconducteur. Cet octaèdre de coordination est par contre symétrique (6.3 liaisons de longueurs très voisines) dans l'antimoine liquide; la distorsion de Peierls locale s'est évanouie. Le bismuth liquide à une coordinence moyenne de 8.8 et ces deux éléments sont métalliques en phase liquide.

L'ordre local des alliages binaires d'arsenic et d'antimoine $\mathrm{As}_{\mathrm{x}} \mathrm{Sb}_{1-\mathrm{x}}$ liquides a aussi été étudié par diffraction de neutrons. Le nombre de coordination $\mathrm{Z}$ et les distances interatomiques sont analysés en termes de la concentration en arsenic; $Z$ augmente continûment entre une valeur de 3.5 pour l'arsenic pur et de 6.3 pour l'antimoine pur. Cela correspond à une valeur effective de p/q qui crô̂t continûment de 2.7 (As) à 4 (Sb).

A ce stade, il faut expliquer pourquoi certains éléments (et alliages) (p. ex. As) gardent leur distorsion de Peierls à basse température tandis que d'autres (p. ex. Sb) la voient disparaître. Un modèle de liaisons fortes (ou Hückel étendu, voir §1.1) simplifié (atomes moyens) en vue de l'application aux composés binaires, montre que la variation du seul paramètre de dureté p (4) entre les valeurs que celui-ci prend pour $\mathrm{As}$ et $\mathrm{Sb}$, permet de reproduire l'ensemble du comportement du système $\mathrm{As}_{x} \mathrm{Sb}_{1-\mathrm{x}}$ dans la phase liquide [3]. 
NEUTRONS ET SYSTÈMES DÉSORDONNÉS

\begin{tabular}{|c|c|c|c|c|}
\hline & $\mathrm{q}_{2} / \mathrm{q}_{1}$ & $\mathrm{Z}_{\text {liquide }}$ & $\Delta \mathrm{V} / \mathrm{V}$ & $\begin{array}{c}\mathrm{S}_{\text {fusion }} \\
\text { cal/K/môle }\end{array}$ \\
\hline $\mathrm{Li}$ & 1.83 & 9.5 & 5 & $2-3$ \\
\hline $\mathrm{Na}$ & 1.85 & 10.4 & 2.5 & 1.7 \\
\hline $\mathrm{Mg}$ & 1.82 & 10.9 & 4.1 & 2.3 \\
\hline $\mathrm{Al}$ & 1.85 & 11.5 & 6.4 & 2.7 \\
\hline $\mathrm{Fe}$ & 1.83 & 10.6 & 3.6 & 2.0 \\
\hline $\mathrm{Cu}$ & 1.82 & 11.3 & 4.2 & 2.3 \\
\hline $\mathrm{Si}$ & 2.07 & 6.4 & -10 & 7.1 \\
\hline $\mathrm{Ge}$ & 2.00 & 6.8 & -4.8 & 7 \\
\hline $\mathrm{As}$ & 1.53 & 3.5 & 10 & - \\
\hline $\mathrm{Sb}$ & 1.96 & 6.3 & 1 & 5.3 \\
\hline $\mathrm{Bi}$ & 1.95 & 8.8 & -3.3 & - \\
\hline $\mathrm{GaAs}$ & 2.1 & 5.5 & -11 & 4.7 \\
\hline $\mathrm{InSb}$ & 2.1 & 6.3 & -3.7 & 7.1 \\
\hline $\mathrm{ZnTe}$ & 1.70 & 3.7 & 8.0 & 3.9 \\
\hline $\mathrm{CdTe}$ & 1.72 & 3.7 & 0.7 & 3.8 \\
\hline $\mathrm{HgTe}$ & 2.11 & 6.3 & -3.3 & 4.5 \\
\hline $\begin{array}{c}\text { Connel- } \\
\mathrm{Temkin}\end{array}$ & 1.74 & 4 & - & - \\
\hline $\begin{array}{c}\mathrm{Sphères} \\
\text { dures }\end{array}$ & 1.86 & $>10$ & - & 13.5 \\
\hline & & & & \\
\hline
\end{tabular}

Tableau 6. Valeurs de quelques paramètres pour les éléments et composés au point de fusion. $q_{1}$ et $\mathrm{q}_{2}$ sont les positions des deux premiers maxima du facteur de structure du liquide. $\Delta \mathrm{V}$ est la variation de volume à la fusion. $S_{\text {fusion }}$ est l'entropie de fusion.

En conclusion, on voit que la distorsion subsiste dans le liquide lorsque l'énergie thermique est inférieure à l'énergie de la distorsion $\Delta \mathrm{E}=\mathrm{E}$ (cubique simple) - $\mathrm{E}(\mathrm{A} 7)$. Tout s'explique par une simple compétition entre le terme enthalpique et le terme entropique (cf. tableau 7). 
S4⿻

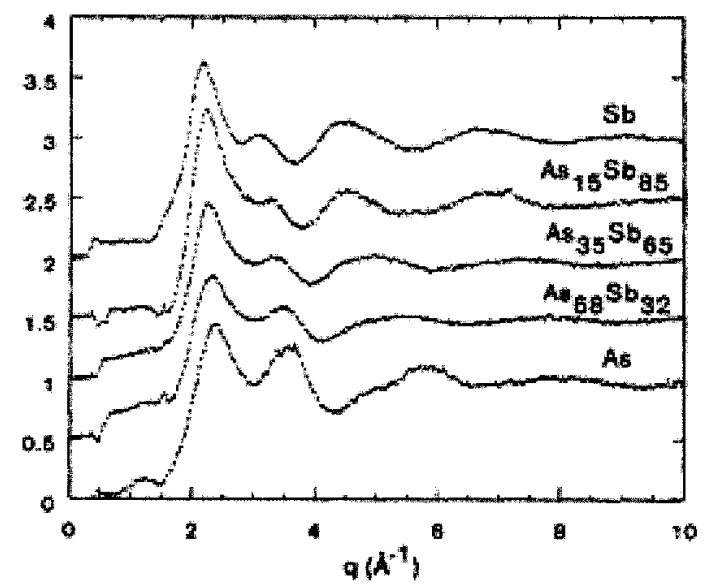

Figure 6. Facteurs de structure $\mathrm{S}(\mathrm{q})$ de $\mathrm{As}_{\mathrm{x}} \mathrm{Sb}_{1-\mathrm{x}}$, avec $\mathrm{x}=0 ., 0.15,0.35,0.68$ et 1.00 . mesurés juste au-dessus du point de fusion, soit respectivement à $933 \mathrm{~K}, 973 \mathrm{~K}, 973 \mathrm{~K}, 1023 \mathrm{~K}$ et $1098 \mathrm{~K}$.

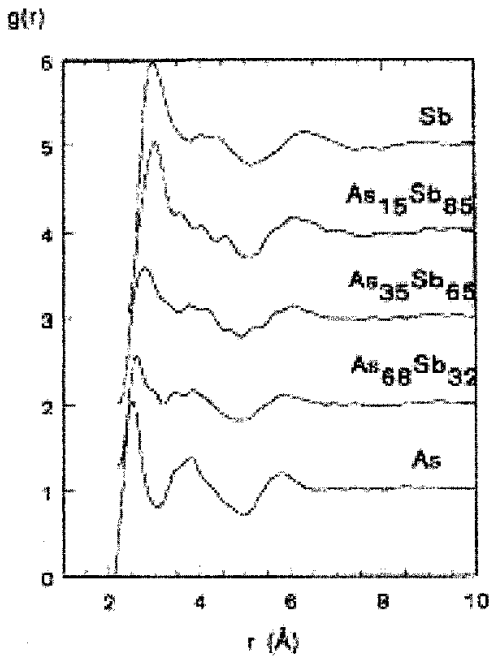

Figure 7. Fonctions de corrélation de paires $\mathrm{d}^{\prime} \mathrm{As}_{\mathrm{x}} \mathrm{Sb}_{1-x}$, avec $\mathrm{x}=0 ., 0.15,0.35,0.68$ et 1.00 . mesurées juste au-dessus du point de fusion, soit respectivement à $933 \mathrm{~K}, 973 \mathrm{~K}, 973 \mathrm{~K}, 1023 \mathrm{~K}$ et $1098 \mathrm{~K}$. Le pic des seconds voisins de l'As (à $3.1 \AA$ dans le solide) est invisible puis le pic se précise lorsque la distorsion diminue. Pour le Sb, il est confondu avec le pic des premiers voisins. 


\begin{tabular}{lcccccl}
\hline & $\mathrm{r}_{1} / \mathrm{r}_{\mathrm{c}}$ & $\begin{array}{c}\mathrm{T}_{\text {fusion }} \\
\mathrm{K}\end{array}$ & $\begin{array}{c}\Delta \mathrm{E} \\
\mathrm{eV}\end{array}$ & $\Delta \mathrm{E} / \mathrm{kT}_{\mathrm{f}}$ & $\mathrm{p}$ & dist. Peierls \\
\hline $\mathrm{As}$ & 1.25 & 1090 & .15 & 1.5 & 5.4 & distordu \\
$\mathrm{Sb}$ & 1.17 & 904 & .03 & 0.4 & 8.0 & pas distordu \\
$\mathrm{Bi}$ & 1.12 & 544 & .004 & 0.1 & 9.6 & pas distordu \\
\hline
\end{tabular}

Tableau 7. Taux de distorsion de Peierls, température de fusion, énergie de distorsion de Peierls $\left(\Delta \mathrm{E}=\mathrm{E}_{\text {distordu }}-\mathrm{E}_{\mathrm{non} \text { distordu }}\right)$ pour les éléments de la colonne $\mathrm{V}$ et paramètre $\mathrm{p}$ de la répulsion. On voit que plus p est élévé, plus la distorsion de Peierls est faible et plus aisément elle disparaît avec la température.

Les éléments et composés du groupe VI présentent les mêmes tendances. Le sélénium fond en gardant sa coordinence $Z=2$ tandis que le tellure, moins déformé, voit sa coordinence passer de 2 à 2.7 lors de la fusion. La distorsion de Peierls est encore partiellement présente. Le polonium, quant à lui, ne présente pas de distorsion de Peierls à température ambiante. Cette évolution du comportement est également pilotée par le paramètre de répulsion p.

\subsection{La fusion des composés IV-VI}

Ces composés, isoélectroniques des précédents avec 5 électrons $\mathrm{s}$ et $\mathrm{p}$ en moyenne par atome, on fait l'objet de nombreuses études récentes. Ils sont intéressants au plan technologique car ils sont proches des composés ternaires GeSbTe qui constituent les matériaux de stockage de l'information dans les CD et DVD réinscriptibles. Ils montrent des comportements intéressants et spectaculaires du point de vue structural comme nous allons le montrer.
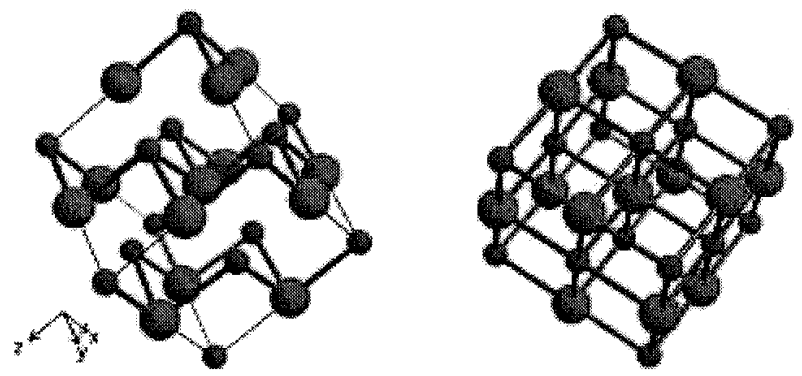

Figure 8. Structure déformée de $\mathrm{GeTe}$ à basse température $\left(\mathrm{T}<432^{\circ} \mathrm{C}\right)$ et sa structure $\mathrm{NaCl}$ à haute température $\left(\mathrm{T}>432^{\circ} \mathrm{C}\right)$

La plupart des composés IV-VI sont des déformations de la structure $\mathrm{NaCl}$ (fig. 8), du groupe $\mathrm{R} 3 \mathrm{~m}$ $\left(\alpha=88.17^{\circ}\right)$, de coordinence $Z=3(+3)$. Pour GeTe à basse température, la distance de premiers voisins est $r_{1}=2.84 \AA$ et le taux de distorsion est $r_{2} / r_{1}=1.11$. Au-delà de $432^{\circ} \mathrm{C}$, GeTe adopte la 
structure $\mathrm{CFC}$ du $\mathrm{NaCl}$, donc non distordue. On pourrait raisonnablement penser que l'histoire s'arrête ici mais il n'en est rien. Dans la phase liquide, le GeTe est de nouveau distordu, mais d'une autre manière car le volume supplémentaire qu'il a acquis lors de la fusion lui permet de retrouver une distorsion. On a donc une transition de phase réentrante distordu-non distordu-distordu.
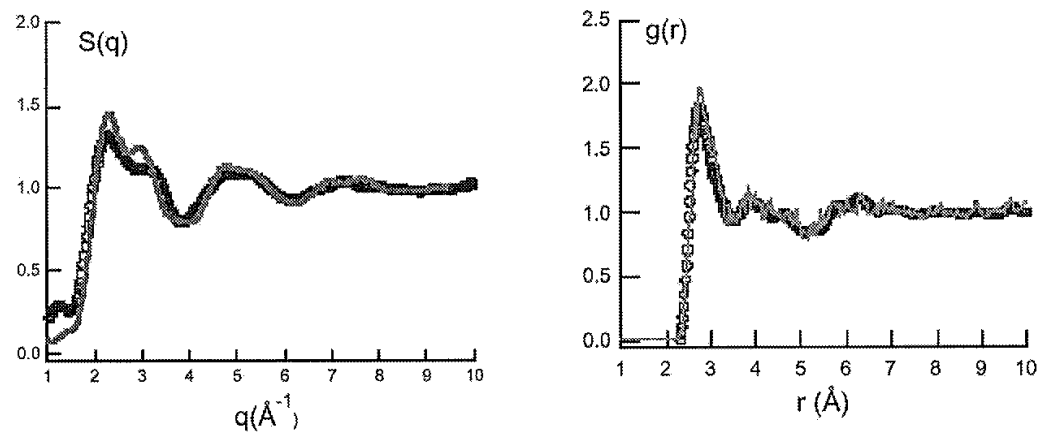

Figure 9. Spectres de diffraction et fonctions de corrélation de paires de GeTe, expérimentaux (cercles, $900^{\circ} \mathrm{C}$ ) et simulés sur ordinateur (dynamique moléculaire ab initio).

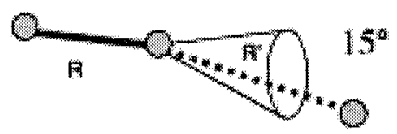

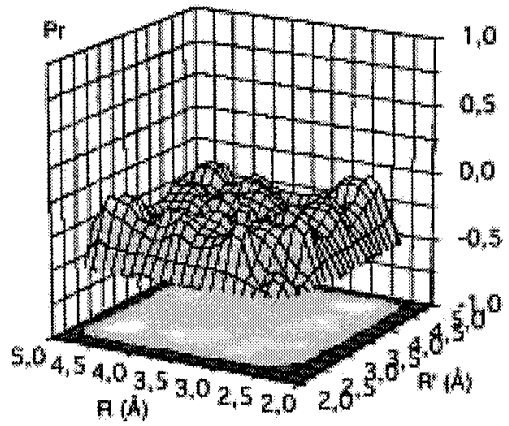

$T=6000 \mathrm{~K}$

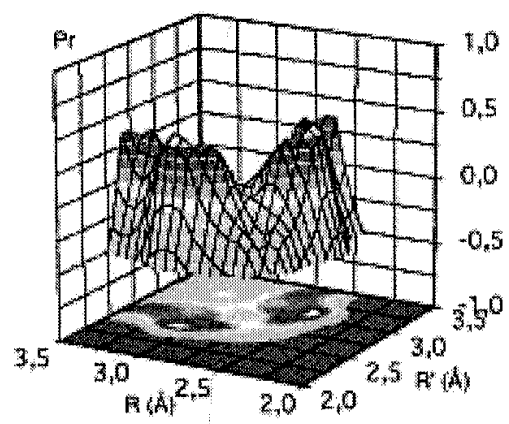

$\mathrm{T}=1000 \mathrm{~K}$

Figure 10. Fonctions de corrélation à 3 corps "presque alignés" obtenues par dynamique moléculaire ab initio. On voit que, à $1000 \mathrm{~K}$ (juste au-dessus de $\mathrm{T}_{\mathrm{f}}$ ), la probabilité de l'alternance courte-longue est élevée. Par contre, à $6000 \mathrm{~K}$, la distribution des distances est assez uniforme. 
Afin d'analyser ce qui se passe au niveau microscopique, on réalise des "expériences numériques" de dynamique moléculaire $a b$ initio. Après avoir vérifié que le résultat de la simulation reproduit bien le spectre expérimental, tant dans l'espace direct que dans l'espace réciproque (fig. 9), on analyse les environnements locaux, obtenus par dynamique moléculaire, aux plans de l'ordre topologique et de l'ordre chimique. Pour ce faire, nous prenons deux atomes premiers voisins A et $\mathrm{B}$ et nous recherchons les atomes $\mathrm{C}$, premiers voisins situés dans un cône de petite ouverture $\mathrm{p}$. ex. $15^{\circ}$ (figure 10 ).

On représente la probabilité d'avoir deux distances $\operatorname{Pr}\left(R, R^{\prime}\right)$ entre trois atomes "presque alignés". On voit que, à $1000 \mathrm{~K}$, la probabilité de l'alternance courte-longue est élevée comme en témoigne l'existence de pics. Par contre, à $6000 \mathrm{~K}$, la distribution des distances est assez uniforme puisque la fonction $\operatorname{Pr}\left(R, R^{\prime}\right)$ présente un plateau. On a ainsi démontré que la distorsion de Peierls existait encore dans le liquide, pour autant que le terme entropique ne soit pas trop élevé.

La simulation permet également d'avoir accès à l'ordre chimique, que l'on ne pourrait atteindre en diffraction de neutrons que par la substitution isotopique. Toutefois un traitement en formalisme de Bathia-Thornton pourrait permettre de comparer expérience et théorie sur l'ordre chimique.
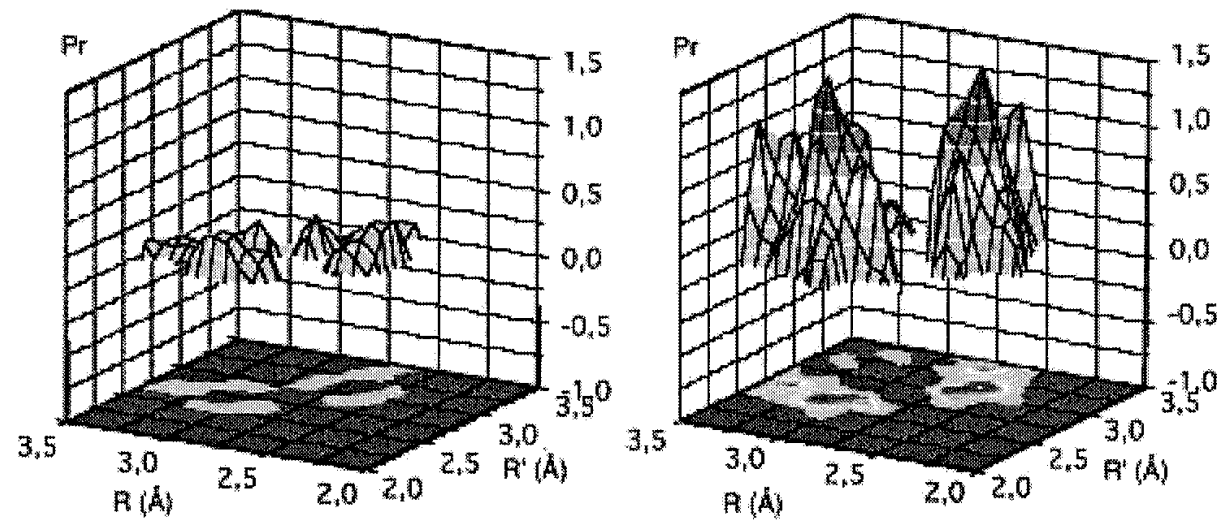

Figure 11. Fonctions de corrélation à 3 corps "presque alignés" obtenus pour GeTe à $1000 \mathrm{~K}$ (juste au-dessus de $\mathrm{T}_{\mathrm{f}}$ ) par dynamique moléculaire $a b$ initio. A gauche, triplets $\mathrm{Ge}-\mathrm{Ge}-\mathrm{Ge}$, à droite, triplets $\mathrm{Ge}-\mathrm{Te}-\mathrm{Ge}$. On voit que la corrélation liaison courte-liaison longue (presque alignées) est très importante pour les liaisons hétéropolaires mais négligeable pour les liaisons homopolaires.

La simulation nous apprend donc que l'on a une double transition de phase réentrante distordunon distordu-distordu-non distordu en fonction de la température.

\section{LES EFFETS DE LA PRESSION}

La pression a pour effet de réduire, puis d'annihiler la distorsion de Peierls. On constate que pour l'arsenic, la transition de la phase $\mathrm{A} 7$ au cubique simple se produit à haute pression (25 $\mathrm{GPa})$, pour le $\mathrm{Sb}$ à $8 \mathrm{GPä} \mathrm{et} \mathrm{pour} \mathrm{le} \mathrm{Bi}$ à $2.5 \mathrm{GPa}$. Ceci est compatible avec l'augmentation de la dureté du potentiel répulsif lorsque le nombre atomique augmente. Les éléments les plus lourds ont des distorsions plus faibles et donc transitent à plus basse pression et à plus basse température. 
D'une manière générale, dans les semiconducteurs, l'effet de la pression produit les mêmes effets que la température [21] alors que c'est l'inverse pour les métaux. Dans les semiconducteurs, lors de la fusion, la coordinence augmente en général (ainsi que la distance interatomique), de même que lorsqu'ils subissent une transition de phase sous l'effet de la pression.

\section{EXTENSIONS : $\mathrm{H}_{2}, \mathrm{H}_{2}$ O ET METAUX DILATES}

La physique que nous avons développée ici est bien plus générale que les différents cas que nous avons présentés. Signalons en particulier les effets de pression, étudiés récemment, qui conduisent à une disparition de la brisure de symétrie. Mentionnons quelques cas importants:

- la métallisation de l'hydrogène sous pressions extrêmes. On espère mettre en évidence l'hydrogène atomique métallique au-delà de $400 \mathrm{GPa}$.

- La symétrisation observée de la liaison hydrogène O-H-O dans la glace X [15]

- La transition métal-isolant dans le sodium dilaté (atomes Na dans un clathrate) [16].

Tous ces exemples montrent l'universalité de phénomènes, en apparence différents, dans le champ de la physique. Or la recherche de l'universalité est un des buts de la physique. La diffraction des neutrons y a contribué largement.

\section{CONCLUSIONS}

L'étude des liquides par diffraction de neutrons, combinée avec d'autres techniques et une bonne modélisation de la liaison chimique, a permis de dégager des règles de comportement systématiques dans le domaine des liquides covalents. Nous avons repris ici quelques cas exemplatifs qui nous ont permis de démontrer le caractère local de la liaison chimique. La périodicité, si elle permet de simplifier la description des solides cristallins, n'influence que peu les propriétés structurales. En particulier l'instabilité électronique de Peierls, et la règle de l'octet qui sen résulte, continuent à exister dans le liquide, pour autant que le terme entropique n'efface pas leur présence.

\section{RÉFÉRENCES}

1. J.K. Burdett. Chemical Bonding in Solids. Oxford University Press, New-York, (1995).

2. J.-Y. Raty, thèse de doctorat, Université de Liège (B), 1999 (non publiée)

3. J.-P. Gaspard, A. Pellegatti, F. Marinelli, C. Bichara, Phil. Mag. (3), 727-744 (1998).

4. R. Peierls. Quantum Theory of Solids. Pergamon, Oxford, (1955).

5. W. A. Harrison. Electronic Structure and the Properties of Solids. W. H. Freeman, San Francisco, (1980).

6. J.-P. Poirier Intraduction to the Physics of the Earth's Interior 2nd Edition: Cambridge University Press, 2000

7. R. Bellissent, G. Tourand, J. de Phys. 37, 1423 (1976).

8. J.-P. Ambroise, R. Bellissent, Rev. Phys. Appl., 19, 731 (1984)

9. D. Chandler Introduction to Modern Statistical Mechanics, Oxford University Press (1987)

D. Frenkel, Smith, Understanding Molecular Simulation, Academic Press (1996)

10. J.P. Hansen, I.R. McDonald. Theory of Simple Liquids. 2nd Edition. Academic Press, London. (1986);

M. P. Allen et D. J. Tildesley, Computer simulation of liquids Clarendon Press Oxford (1987);

F. Ercolessi, A molecular dynamics primer, ICTP Trieste (1997) http://www.sissa.it/furio 
11. V.M. Glazov, S.N. Chizhevskaya, N.N. Glagoleva. Liquid semiconductors, Plenum, NewYork, (1969).

12. A. SanMiguel. Etudes structurales de $\mathrm{Zn} T$ Te, HgTe et $\mathrm{Z}_{n} x H g_{l_{-x}}$ Te sous pression. thèse de doctorat, Paris VI (Fr.), (1993) (non publiée).

13. R. Bellissent, C. Bergman, R. Céolin, J.-P. Gaspard, Phys. Rev. Letters, 59 (6), 661 (1987);

C. Bergman, A. Pellegatti, R. Bellissent, A. Menelle, R. Céolin, J.-P. Gaspard, Physica B156-157, 158 (1989)

14. J. Hafner, Phys. Rev. Lett. 62 (7), 784 (1989).

15. http://www.esrf.fr/info/science/highlights/97-98/PF79.htm

16. F. Brunet, P. Melinon, A. San Miguel, A. Perez, A.-M. Flank, E Reny, C. Cros , M. Pouchard, Phys. Rev. B, 61, 16550 (2000)

17. J R Chelikowsky, Jeffrey J Derby, V. V Godlevsky, M. Jain, J Y Raty, J. Phys.: Condens. Matter 13 R817-R854 (2001)

18. F. Ducastelle, J. de Physique, 31 1055-1062 (1970)

19. H. Fischer, communication privée.

20. P. Chieux, Journ. of Molecular Structure, 296, 177 (1993)

21. Mujica, Angel Rubio, A. Munoz, and R. J. Needs, Review of Modern Physics (2003), in print. 